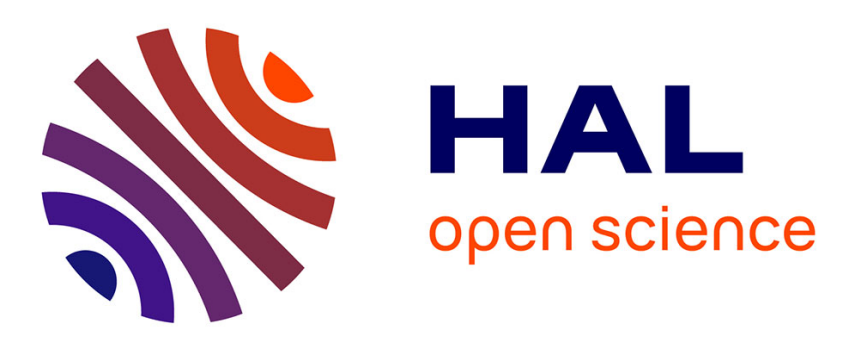

\title{
Lateral Porous Silicon Interferometric Transducer for Sensing Applications
}

Yingning He, Douglas Silva de Vasconcellos, Véronique Bardinal, David

Bourrier, Éric Imbernon, Ludovic Salvagnac, Adrian Laborde, Xavier Dollat, Thierry Leichle

\section{To cite this version:}

Yingning He, Douglas Silva de Vasconcellos, Véronique Bardinal, David Bourrier, Éric Imbernon, et al. Lateral Porous Silicon Interferometric Transducer for Sensing Applications. 2018 IEEE SENSORS, Oct 2018, New Delhi, India. 10.1109/ICSENS.2018.8589773 . hal-03212723

\section{HAL Id: hal-03212723 \\ https://hal.science/hal-03212723}

Submitted on 29 Apr 2021

HAL is a multi-disciplinary open access archive for the deposit and dissemination of scientific research documents, whether they are published or not. The documents may come from teaching and research institutions in France or abroad, or from public or private research centers.
L'archive ouverte pluridisciplinaire $\mathbf{H A L}$, est destinée au dépôt et à la diffusion de documents scientifiques de niveau recherche, publiés ou non, émanant des établissements d'enseignement et de recherche français ou étrangers, des laboratoires publics ou privés. 


\title{
Lateral Porous Silicon Interferometric Transducer for Sensing Applications
}

\author{
Yingning $\mathrm{He}^{1,2}$, Douglas Silva de Vasconcellos ${ }^{1}$, Véronique Bardinal ${ }^{1}$, David Bourrier ${ }^{1}$, Eric Imbernon ${ }^{1}$, Ludovic \\ Salvagnac ${ }^{1}$, Adrian Laborde ${ }^{1}$, Xavier Dollat ${ }^{1}$, and Thierry Leichlé ${ }^{1}$ \\ ${ }^{1}$ LAAS-CNRS, Université de Toulouse, CNRS, Toulouse, France \\ ${ }^{2}$ State Key Laboratory of Molecular Engineering of Polymers, Department of Macromolecular Science, Fudan University, \\ Shanghai, China \\ tleichle@1aas.fr
}

\begin{abstract}
In this work, we demonstrate the transducing ability of lateral porous silicon membranes (LPSi) using optical interferometry. To this aim, we carry out Fourier-transform infrared spectroscopy measurements using a microscope equipped with an appropriate objective in order to overcome the difficulty to obtain interference signal from LPSi membranes with small dimensions. Reflectance spectra are recorded while filling the membrane with various solvents and their analysis provide estimations of the effective optical thicknesses and the resulting index of refraction of the fillers in the NIR range. The results show that the various solvents can appropriately be identified and discriminated through the derived refractive indices, thus providing a proof-of-concept of the LPSi interferometric transducer.
\end{abstract}

Keywords—porous silicon; interferometry; lab on a chip; biosensor

\section{INTRODUCTION}

Sample analysis usually involves the detection of a specific biochemical compound. This is achieved by transducing a biological recognition event into a measurable and quantifiable signal. Common signal transducers include electrical, electrochemical, optical, mechanical, and thermal sensors. The optical sensing scheme enables to probe surfaces and films in a nondestructive manner; it offers advantages in speed, sensitivity, and robustness, as well as permitting in situ sensing and real-time measurements. Out of the various materials available to constitute optical-based biosensors, porous silicon has high surface area, convenient surface chemistry, and has been widely studied in interferometric sensing configuration [1].

Porous silicon-based interferometric sensors and biosensors consist of vertical porous silicon layers created into a silicon wafer where the pores are oriented perpendicular to the wafer surface [2]. Vertical porous silicon is most often created by silicon anodization. For biosensing purpose, the surface of the porous silicon is modified with biomolecules. Reflection of white light at the top and bottom of the porous silicon layer results in an interference pattern (Fabry-Pérot fringes) that depends on the refractive index of the porous silicon matrix. Interactions of molecular species with their recognition partners immobilized onto the porous silicon surface induce a change in the refractive index of the porous layer, giving rise to a wavelength shift in the fringe pattern.
It is possible to carry out biosensing using porous silicon via optical measurement through a fluidic cell, as demonstrated by works where vertical porous silicon is integrated into microfluidics for real-time detection [3]. These structures have been vastly studied for biosensing of various elements, e.g. DNA, protein, enzyme activity and bacteria [4].

In a previous work [5], we have developed a unique process to fabricate porous silicon membranes with lateral pores, i.e. with horizontal as opposed to above described vertical pores, and integrate them into planar fluidic channels. While the use of porous layers with vertical pores for biosensing in two-dimensional microfluidics results in flow-over (FO) sensing configurations, porous silicon membranes with lateral pores enable liquid to flow through (FT) (Figure 1). Theoretical works have shown that, with similar sensor footprints, flow-through (FT) sensor offers up to 20-fold improvement in response time [6]. Besides, the experimental comparison between FO and FT sensors, later investigated using porous silicon microcavities and freestanding membranes [7], revealed some issues encountered with FO sensors where analytes or contaminants can be trapped within the closed-ended pores. Thus, following our previous results on fabricated lateral porous silicon (LPSi) membranes, the aim of the present work is to demonstrate the sensing ability of lateral porous silicon via interferometric measurements to offer solutions for future on-chip flowthrough biosensors.

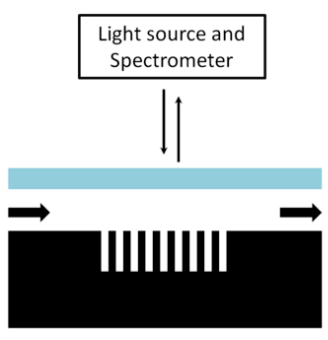

(a)

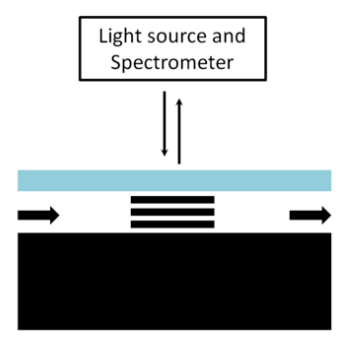

(b)
Fig. 1. Two interferometric sensing configurations with porous silicon: (a) a typical flow-over configuration (FO) with a surface-based sensor within a microfluidic channel, (b) a flow-through configuration (FT) with an array of lateral nanopore tubes adequately connecting both microchannels. 


\section{MATERIALS AND METHODS}

Optical measurements were performed on a VERTEX 70 Fourier Transform Infra-Red spectrometer (FTIR) (Bruker Optics) equipped with a tungsten light source, a Quartz beam splitter (T502) and a Si-diode detector (SiD 510), which were used to cover a spectral range from 600 to $1100 \mathrm{~nm}$. The spectrometer was connected to a HYPERION microscope stage holding various objectives and apertures. A $36 \times$ objective was used for localized measurements.

Microfluidic chips bearing lateral porous silicon membranes were fabricated following the process described in [5]. Each $1.6 \mathrm{~cm} \times 1.6 \mathrm{~cm}$ microfluidic chip integrated two $5 \mu \mathrm{m}$ deep microchannels separated by a $10 \mu \mathrm{m}$ thick and 4 $\mu \mathrm{m}$ high porous silicon membrane with average porous diameter of $\sim 15 \mathrm{~nm}$. The porous membrane was obtained through silicon anodization at $200 \mathrm{~mA} \mathrm{~cm}$ during $1 \mathrm{~min}$. The porous silicon chip was loaded into a sample holder which was subsequently fixed on the microscope stage (Figure 2). The sample holder was connected to a 4-channel reservoir (FLUIWELLTM, Fluigent) with pressure sources (MFCS-8C, Fluigent) to control the fluid flow inside the chip.

Various solvents loaded in the reservoir were pumped into the microchannels and subsequently pushed through the LPSi membrane via positive pressure to ensure the full infiltration of the analyte into the membrane. The observation window was downsized to a rectangular area with dimensions of $10 \times 100 \mu \mathrm{m}^{2}$ using adjustable $\mathrm{x}$ - and $\mathrm{y}$ apertures. This window size was chosen so as to only observe the $10 \times 250 \mu \mathrm{m}^{2}$ LPSi membrane. Reflectance data acquisition was carried out with a spectral resolution of 4 $\mathrm{cm}^{-1}$. In order to test the interferometric transduction capability of the LPSi membranes, three kinds of solvents (water, ethanol, acetone) were tested. It is important to note that, prior to the measurements, a reference spectrum was taken onto a gold mirror using the same experimental conditions (i.e. sample stage height and observation window size). Besides, before injecting test solutions, the chip was exposed to oxygen plasma in order to increase the wettability of the microchannels and the nanopores.

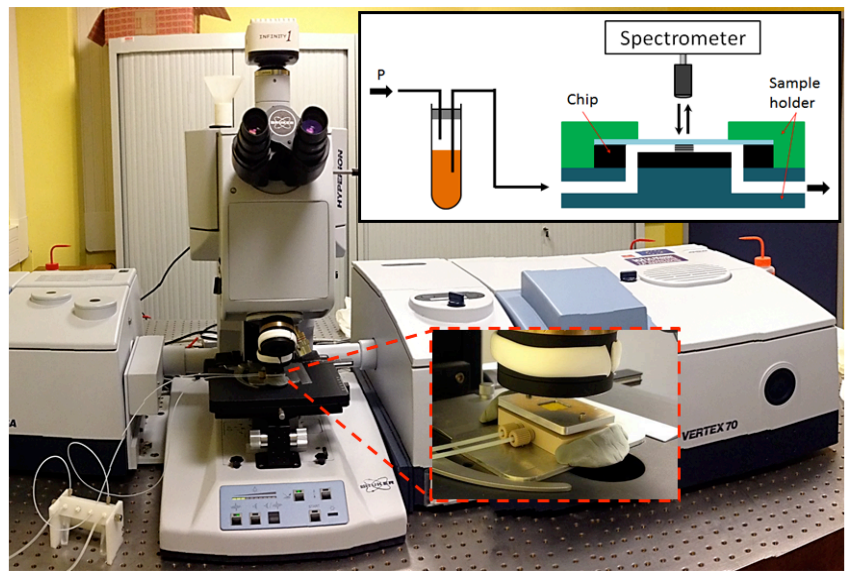

Fig. 2. Experimental setup for microscale analysis and fluid-managing system used for optical detection.

\section{RESULTS AND DISCUSSIONS}

\section{A. Validation of Optical Transduction}

The relationship between the optical path length $2 n L \cos \theta$, also referred to as the effective optical thickness (EOT), and the Fabry-Pérot fringes [8] is:

$$
2 n L \cos \theta=m \lambda_{\max }
$$

Where, $n$ and $L$ are the refractive index and the thickness of the PSi layer, $\theta$ is the incident angle of the light at the $\mathrm{PSi} / \mathrm{Si}$ interface, $m$ is an integer corresponding to the spectral order of the fringe and $\lambda_{\max }$ is the wavelength of the fringe maximum.

Since the refractive index of porous silicon membranes depends on the pore-filling medium, we have tested the ability of our platform to detect a change of refractive index through the effective optical thickness by loading the membranes with liquids of various optical properties. Evaporable solvents (water, ethanol and acetone) were tested according to the procedure described in section II. Figure 3 presents the resulting reflectance spectra. Compared to empty and dried porous silicon, the presence of all three liquids clearly led to a red shift of the spectrum corresponding to an increase in refractive index. The wavelength shifts from bare porous silicon are in the following order: water $(n \sim 1.328)<$ acetone $\approx$ ethanol $(n \sim 1.35)$.

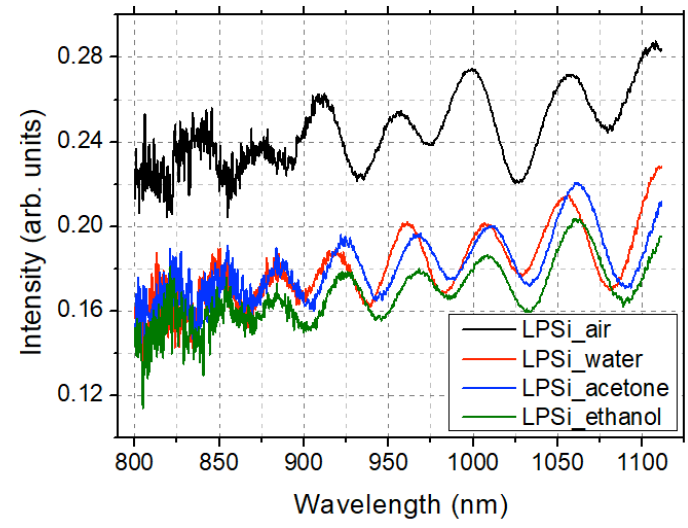

Fig. 3. Experimental reflectance spectra of a LPSi membrane filled with different solvents: water, acetone, and ethanol. The spectrum marked as air means that no liquid fills the pores.

\section{B. Analysis of Reflectance Spectra}

We have analyzed the Fabry-Pérot fringes by means of two methods in order to derive the measured effective optical thickness.

First, we have plotted the spectral order of each fringe (peak number) as a function of frequency, as shown in Figure 4 , where the slope of the linear fit equals the effective optical thickness $2 n L \cos \theta$ [9]. Since the membrane thickness $(L)$ can be measured by SEM and $\theta$ is given by the numerical aperture of the objective, we can derive an experimental value for the refractive index. 


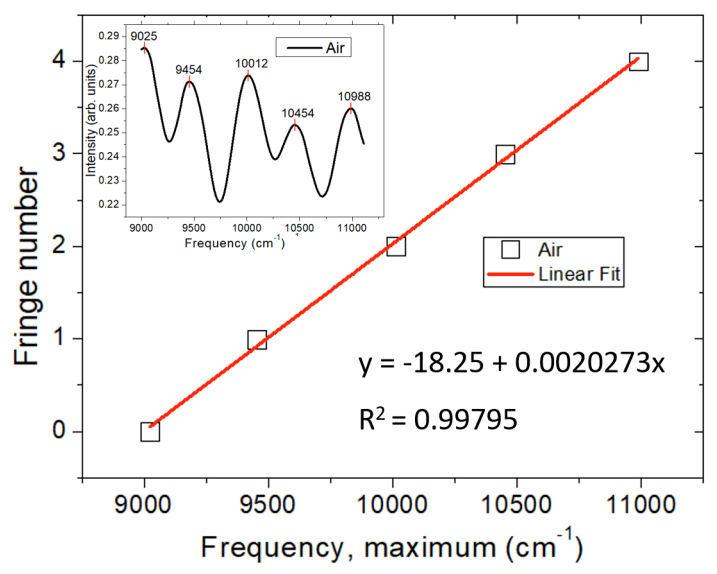

Fig. 4. Fringe number as a function of corresponding spatial frequency. The inset shows the spectrum replotted in frequency unit and the peaks of the interference pattern corresponding to successive peak number.

The spectrum analysis was also carried out by Reflectometric Interference Fourier Transform Spectroscopy (RIFTS) [9]. This method computes the frequency spectrum of an input waveform by fast Fourier transform, yielding a peak whose position along the $\mathrm{x}$-axis corresponds to the effective optical thickness.

The theoretical refractive index of the porous silicon layer can be estimated by Bruggeman Effective Medium Model:

$$
P \frac{n_{\text {fill }}^{2}-n_{\text {layer }}^{2}}{n_{\text {fill }}^{2}+n_{\text {layer }}^{2}}+(1-P) \frac{n_{\text {skelenton }}^{2}-n_{\text {layer }}^{2}}{n_{\text {skelenton }}^{2}+2 n_{\text {layer }}^{2}}=0
$$

Where $P$ is the porosity of porous silicon $(40 \%$ in the case of the fabricated LPSi membranes), $n_{\text {fil }}$ is the refractive index of the medium filling the pores, $n_{\text {skeleton }}$ is the refractive index of the porous material (i.e. the refractive index of silicon, 3.673 [10]) and $n_{\text {layer }}$ is the refractive index of the composite porous silicon.

TABLE I. EXPERIMENTAL AND THEORETICAL INDICES OF REFRACTION OF LATERAL POROUS SILICON MEMBRANES FILLED WITH VARIOUS SOVENTS

\begin{tabular}{|c|c|c|c|c|c|}
\hline Medium & & Air & Water & Acetone & Ethanol \\
\hline \multirow{2}{*}{$\begin{array}{c}\text { Expethod } \\
\text { (RIFTS) }\end{array}$} & EOT $(\mathrm{nm})$ & $20255 \pm 158$ & $21679 \pm 158$ & $21679 \pm 158$ & $21679 \pm 158$ \\
\cline { 2 - 6 } & $n_{\text {layer }}$ & $2.56 \pm 0.020$ & $2.74 \pm 0.020$ & $2.74 \pm 0.020$ & $2.74 \pm 0.020$ \\
\hline \multirow{2}{*}{$\begin{array}{c}\text { Experimental } \\
\text { (fitting) }\end{array}$} & EOT $(\mathrm{nm})$ & $20273 \pm 459$ & $21293 \pm 216$ & $21386 \pm 156$ & $21394 \pm 392$ \\
\cline { 2 - 6 } & $n_{\text {layer }}$ & $2.54 \pm 0.058$ & $2.67 \pm 0.027$ & $2.68 \pm 0.020$ & $2.68 \pm 0.049$ \\
\hline $\begin{array}{c}\text { Theoretical } \\
\text { approximation }\end{array}$ & $n_{\text {fill }}$ & 1 & 1,328 & 1,354 & 1,357 \\
\cline { 2 - 6 } & $n_{\text {layer }}$ & 2.56 & 2.69 & 2.70 & 2.70 \\
\hline
\end{tabular}

Table 1 provides the refractive indices derived from the experimental methods and from theory. Since the dispersion of the index of refraction is fairly small in the wavelength range considered in this study [9], it was assumed constant for all solvents and the index of porous silicon as a function of filling solvent was obtained using values at $830 \mathrm{~nm}$ and 20 ${ }^{\circ} \mathrm{C}$ [11]. Data show that values obtained from the experimental methods are in accordance with the theoretical ones. The composite refractive index $\left(n_{\text {laver }}\right)$ is in order of air $<$ water $<$ acetone $\approx$ ethanol, which, indeed, corresponds to the shifts of measured interference patterns, thus demonstrating the possibility to discriminate the various solvents with lateral porous silicon.

\section{CONCLUSION}

In this work, we have demonstrated the Fabry-Pérot interferometric transduction capability of lateral porous silicon membranes bridging microfluidic channels. The specificity and difficulty of our study is that the LPSi membranes exhibit small dimensions, which increases the difficulty to obtain exploitable interference signals. Nevertheless, this issue was overcome by optimizing the experimental setup and the optical measurement conditions. We were able to identify the presence of solvents with different refractive index, thus providing a proof-of-concept of the LPSi interferometric transducer.

\section{ACKNOWLEDGMENT}

The authors acknowledge the Agence Nationale de la Recherche (ANR-17-CE09-0024-01). This work was partly supported by the French RENATECH network.

\section{REFERENCES}

[1] Harraz, F. A., "Porous silicon chemical sensors and biosensors: A review." Sensors and Actuators B: Chemical 202 (2014): 897-912.

[2] Dhanekar, S., and Swati, J. "Porous silicon biosensor: current status." Biosensors and bioelectronics 41 (2013): 54-64.

[3] Janshoff, A., Dancil, K. P. S., Steinem, C., Greiner, D. P., Lin, V. S. Y., Gurtner, C., Ghadiri, M. R. "Macroporous p-type silicon FabryPerot layers. Fabrication, characterization, and applications in biosensing." Journal of the American Chemical Society 120.46 (1998): 12108-12116.

[4] Canham, L., "Porous Silicon Optical Biosensors" in Handbook of porous silicon. Springer. 2014

[5] He Y., and Leïchlé T. "Fabrication of lateral porous silicon membranes for planar microfluidics by means of ion implantation." Sensors and Actuators B: Chemical 239 (2017): 628-634.

[6] Escobedo, C., Brolo, A. G., Gordon, R., Sinton, D. "Flow-through vs flow-over: analysis of transport and binding in nanohole array plasmonic biosensors." Analytical chemistry 82.24 (2010): 1001510020.

[7] Kumar, N., Froner, E., Guider, R., Scarpa, M., Bettotti, P. "Investigation of non-specific signals in nanoporous flow-through and flow-over based sensors." Analyst 139.6 (2014): 1345-1349.

[8] Iizuka, K. Elements of Photonics, Vol. 1. John Wiley \& Sons, 2002.

[9] Sailor, M. J. Porous silicon in practice: preparation, characterization and applications. John Wiley \& Sons, 2012.

[10] Aspnes, D. E., and Studna, A. A. "Dielectric functions and optical parameters of $\mathrm{Si}, \mathrm{Ge}, \mathrm{GaP}, \mathrm{GaAs}, \mathrm{GaSb}, \mathrm{InP}, \mathrm{InAs}$, and $\mathrm{InSb}$ from 1.5 to $6.0 \mathrm{eV}$."Physical Review B 27.2 (1983): 985.

[11] Rheims, J., and Wriedt, T. "Refractive-index measurements in the near-IR using an Abbe refractometer." Measurement Science and Technology 8.6 (1997): 601. 